\title{
Zeeman line splitting measurements sampling dense gas in dark cloud and star-forming cores
}

\author{
K. I. Uchida ${ }^{1,3}$, D. Fiebig ${ }^{2}$, and R. Güsten ${ }^{1}$ \\ 1 Max-Planck-Institut für Radioastronomie, Auf dem Hügel 69, 53121 Bonn, Germany \\ 2 Institut für Theoretische Astrophysik, Universität Heidelberg, Tiergartenstraße 15, 69121 Heidelberg, Germany \\ ${ }^{3}$ Cornell University, 108 Space Sciences Building, Ithaca, NY 14853, USA
}

Received 31 October 2000 / Accepted 28 February 2001

\begin{abstract}
We present measurements of Zeeman splitting in SO $J_{\mathrm{N}}=1_{2}-1_{1}$ line emission/absorption arising from dense thermal gas $\left(>10^{6} \mathrm{~cm}^{-3}\right)$ associated with ten compact $\mathrm{H}$ II and star-forming regions. No line-of-sight magnetic field, above an average $1 \sigma$ limit of $0.57 \mathrm{mG}$, is detected toward any of these sources. The W3(OH) region, found in a previous study to have a line-of-sight field of $3 \mathrm{mG}$ in its dense gas, is thus unique in this respect. The implication of this study is that the magnetic fields in dense clouds are supercritical in the sense that they are not capable of supporting their host clouds against gravitational collapse. The relatively low average field limit is consistent with those theories predicting the quick onset of and short timescales for ambipolar diffusion.

Also presented are (1) Zeeman splitting measurements of the CCS $J_{\mathrm{N}}=1_{0}-0_{1}$ line probing the $10^{4-5} \mathrm{~cm}^{-3}$ gas towards three positions in the Taurus star forming region, giving a $1 \sigma$ average upper limit of $66 \mu \mathrm{G}$, and (2) measurements of Zeeman splitting in the $\mathrm{OH}^{2} \Pi_{3 / 2} \quad J=7 / 2 \quad F=3^{+}-3^{-}$hyperfine line toward the compact H II regions DR-21 and G10.62.
\end{abstract}

Key words. radio lines: ISM - magnetic field - H II regions - ISM: molecules - ISM: clouds

\section{Introduction}

Magnetic fields potentially play an important part in the formation and early evolution of stars. Long-wavelength and long-lived MHD waves within molecular clouds may contribute significantly to their slowing against contraction, perhaps enough to account for the observed inefficiency of star formation in the Galaxy. Braking by magnetic fields may be key to how clouds shed their substantial quantities of angular momentum prior to condensing into stars or stellar systems (Basu \& Mouschovias 1994, 1995; Tomisaka et al. 1990). Magnetic fields concievably dictate the size of the subclumps within a cloud and, thus, the masses of the protostellar condensations which ultimately form (Carlberg \& Pudritz 1990). At somewhat later stages a strong field is certain to influence the dynamics and evolution of any accretion disk, outflow, and/or $\mathrm{H}_{\text {II }}$ region which may form around a star (Königl \& Ruden 1993; Pudritz \& Norman 1983, 1986; Blandford \& Payne 1982; Hartmann \& McGregor 1982).

While there exists rather detailed theoretical reasoning in the literature, observational data regarding the strength and structure of magnetic fields are rather poor.

Send offprint requests to: K. Uchida,

e-mail: kuchida@astrosun.tn. cornell.edu
With only a few exceptions (Crutcher et al. 1996b, 1999; Güsten et al. 1994), past Zeeman line splitting studies have characterized $B$ fields only in gas of low to moderate densities - approximately $10^{1-2} \mathrm{~cm}^{-3}$ for $\lambda 21 \mathrm{~cm} \mathrm{Hi} \mathrm{ob-}$ servations, $\sim 10^{3-4} \mathrm{~cm}^{-3}$ (perhaps as high as $10^{5} \mathrm{~cm}^{-3}$ ) for 1665/1667 MHz OH observations. Only recently have measurements in $\sim 10^{6} \mathrm{~cm}^{-3}$ gas been made using the $3 \mathrm{~mm}$ lines of $\mathrm{CN}$ (Crutcher et al. 1996b, 1999). The difficulty with probing higher density condensations, and thus later stages of the star formation process, lies in the fact that while the fields they contain are expected to be stronger than those in the low density material, sensitivity in the measurements is greatly reduced due to the typically higher observing frequencies involved. Very few radicals are known to have all three suitable attributes for use in Zeeman studies: large $g$-factors, high critical densites, and radio transitions that are sufficiently strong. On the other hand, detailing the strength of the field in regions of higher densities is of particular importance; the cloud densities at which supercriticality is achieved, and thus the specifics of the subsequent collapse of the cloud and star forming process, varies greatly between models with $10^{5-6} \mathrm{~cm}^{-3}$ representing the lower bounds of the range (Mouschovias et al. 1985; Nakano 1990). 
Table 1. Molecular parameters

\begin{tabular}{lccl}
\hline Transition & $\nu_{0}[\mathrm{GHz}]$ & $\frac{\Delta \nu_{\mathrm{g}}[\mathrm{Hz}]}{B[\mu \mathrm{G}]}$ & $n\left(\mathrm{~cm}^{-3}\right)$ \\
\hline $\mathrm{CCS}\left(J_{\mathrm{N}}=1_{0}-0_{1}\right)$ & 11.119446 & 0.82 & $1(4-5)$ \\
$\mathrm{SO}\left(J_{\mathrm{N}}=1_{2}-1_{1}\right)$ & 13.044000 & 1.93 & $1(6)$ \\
$\mathrm{OH}\left({ }^{2} \Pi_{3 / 2}, J=\frac{7}{2}, F=3-3\right)$ & 13.434637 & 1.02 & $7(6)$ \\
$\mathrm{OH}\left({ }^{2} \Pi_{3 / 2}, J=\frac{7}{2}, F=4-4\right)$ & 13.441417 & 0.79 & $7(6)$ \\
\hline
\end{tabular}

In this paper we report on a set of extended surveys of Zeeman line splitting, the purpose of which is to detail the strengths of $B$ fields associated with dense $\left(10^{5-6} \mathrm{~cm}^{-3}\right)$ thermal gas and thereby fill a significant gap in the published observational database.

The study was performed with the Effelsberg $100 \mathrm{~m}$ telescope during the last few years by observing

- $\operatorname{SO}\left(J_{\mathrm{N}}=1_{2}-1_{1}\right)$ emission/absorption lines toward ten star-forming and compact $\mathrm{H}$ ir regions;

- $\mathrm{OH}\left({ }^{2} \Pi_{3 / 2}, J=\frac{7}{2}, F=3-3 / 4-4\right)$ absorption towards two compact $\mathrm{H}$ in regions.

While the strong Zeeman coupling of the $\mathrm{SO}\left(J_{\mathrm{N}}=1_{2}-1_{1}\right)$ transition makes it an attractive candidate for performing magnetic field measurements, its linestrength is expected to be weak because of a small spontaneous emission probability $\left(A=2.9710^{-8}\right)$. A large observing aperature, such as the Effelsberg $100 \mathrm{~m}$, is thus the only means of achieving critical sensivities in reasonable integration times.

In addition, we include the results of CCS $\left(J_{\mathrm{N}}=1_{0}-0_{1}\right)$ observations, from the unpublished thesis work of Fiebig (1990), which probes the dense gas $\left(10^{4-5} \mathrm{~cm}^{-3}\right)$ in three low-mass dark cloud cores in the Taurus star forming region.

We discuss the insights these surveys provide with regard to the evolution of magnetic fields in the dense clumps associated with star-forming and young stellar environments and, in particular, to the much argued issue concerning the process of ambipolar diffusion in molecular clouds.

\section{Instrumentation and observations}

The observations were performed during several extended sessions in 1989-90 (CCS) and in 1993-94 (SO and $\mathrm{OH}$ ) with the MPIfR 100-m antenna near Effelsberg, Germany. Both the left and right circularly polarized components were observed simultaneously with dual-channel primefocus receivers and an autocorrelator backend split into $2 \times 512$ channels. The overall system temperature was 40-50 $\mathrm{K}$ at zenith (including antenna and atmospheric losses). Details of the technical set-up are given in Güsten et al. (1994) and Fiebig (1990). The rest frequencies and parameters of the transitions observed are summarized in Table 1; Col. 4 summarizes the approximate $\mathrm{H}_{2}$ critical densities of these transitions.

\section{$\underline{\mathrm{SO} \& \mathrm{OH} \text { observations }}$}

Since only a few astronomical observations of the SO $\left(J_{\mathrm{N}}=1_{2}-1_{1}\right)$ transition had been made prior to this study, short integrations (most of $\sim 3$ min duration) were initially made toward 50 positions of known star-forming regions, $\mathrm{H}$ in regions, and cloud cores to find candidates for the SO $J_{\mathrm{N}}=1_{2}-1_{1}$ Zeeman line splitting measurements (a list of the observations can be requested from the authors). The SO $J_{\mathrm{N}}=1_{2}-1_{1}$ emission was indeed found to be generally weak and was detected in only $\sim 50 \%$ of the sample, with many just at the $2 \sigma$ threshold $(\sim 0.1 \mathrm{~K})$. Of those sources with SO lines suitable for Zeeman splitting measurements, a number of them (Table 2) were mapped to determine their approximate peak position and extent. The positions toward NGC 2071A and VLA 1623 are based on those of SO $J_{\mathrm{N}}=1_{0}-0_{1}(30 \mathrm{MHz})$ emission peaks adjacent to or in the vicinity of prominent CO outflows - they were kindly provided by J. Schmid-Burgk and D. Muders (priv. comm.).

In the absence of a detectable $B$ field, the stronger and narrower the emission/absorption line from a source, the better the attainable upper-limit to the field (Eq. (1)). A total of twelve unique positions were finally selected for deep Zeeman splitting measurements at the $\mathrm{OH}(2)$ and SO (10) transition frequencies (Table 2). Symmetric frequency-switching (by $1 / 4$ of the bandwidth) was used toward all but two of the SO sources. We used either a $3.125 \mathrm{MHz}$ or $6.25 \mathrm{MHz}$ bandpass, thus producing SO spectra with velocity extents of 36 and $74 \mathrm{kms}^{-1}$, respectively, after folding. Asymmetric frequency switching (outside of the observed band) was used toward Sgr B2N and Ori-Kl because the relatively wide lines $(F W H M=$ $\sim 15 \mathrm{~km} \mathrm{~s}^{-1}$ ) from these sources left little or no baseline in the folded symmetrically-switched spectra.

Additionally, the $\mathrm{OH}{ }^{2} \Pi_{3 / 2} \quad J=7 / 2 \quad F=3^{+}-3^{-}$ (13.435 GHz) and $F=4^{+}-4^{-}(13.441 \mathrm{GHz})$ hyperfine transitions were observed toward G10.62 (also one of the SO sources) and toward DR21 OHC. The details of the $\mathrm{OH}$ observational setup are found in Güsten et al. (1994).

Pointing toward a given line-splitting candidate was optimized by performing continuum drift scans across a nearby continuum 3C-source or, in some cases, across the candidate itself (G5.89, G10.62, W3(OH)). Sources 3C 147, 3C 161, 3C 286, and NGC 7027 were used as calibration standards. The beam squint, determined with narrow band $(2 \mathrm{MHz})$ continuum scans across NGC 7027, was 0.2 and $1^{\prime \prime} .2$ at the $\mathrm{SO} J_{\mathrm{N}}=1_{2}-1_{1}$ and $\mathrm{OH}$ ${ }^{2} \Pi_{3 / 2} J=7 / 2$ transition frequencies, respectively, both exceptionally small compared to the FWHM of the antenna beam ( $\left.63^{\prime \prime}\right)$ at $13 \mathrm{GHz}$. The system performance was monitored frequently by observing the characteristic polarization pattern of the $\mathrm{OH}^{2} \Pi_{3 / 2} \quad J=7 / 2 \quad F=4^{+}-4^{-}$ maser line toward $\mathrm{W} 3(\mathrm{OH})$.

\section{CCS observations}

The CCS observations were performed in similar fashion to that of the SO observations. An extended survey was first performed to find potentially suitable sources, the 
selected sources were then mapped and, finally, deep integrations were performed toward their peak positions.

The technical setup was also similar to that for the SO study, with the exception that the dual-channel receivers were located at the secondary focus of the antenna. Symmetric frequency-switching (by $1 / 4$ of the bandwidth) was employed to remove the receiver baseline artifacts. The resolution of all observations was $63 \mathrm{~Hz} /$ channel $\left(0.021 \mathrm{~km} \mathrm{~s}^{-1}\right)$.

Calibration and pointing were accomplished using NGC 7027 and $3 \mathrm{C} 123$. The FWHM beamwidth at the transition frequency of the CCS $J_{\mathrm{N}}=1_{0}-0_{1}$ line is $\left(666^{\prime \prime} 4\right)$. The beam squint $\left(2{ }^{\prime} \cdot 1\right)$ was somewhat larger than that of the SO experiment due to off-axis location of the feed in secondary focus cabin. Unlike the case of the SO receiver at prime focus, where the alignment of the feed to the radio axis of the telescope can be fine tuned (within limits) by tilting and/or rotation of the subreflector, there is no way to perform such fine tuning for a receiver at secondary focus.

\section{Data analysis}

In the presence of a magnetic field, $B$, the magnetic sublevels of a given molecular level $J(\neq 0)$ lose their energy degeneracy. The emission which stem from this splitting have left- and right-circularly polarized components. If the magnetic field is small, but sufficient enough to cause splitting between the components greater than the width of the line itself (as is often the case for maser sources), then the total magnetic field strength may be deduced. If, however, the splitting is less than the width of the line, which is invariably the case for thermally excited gas in H II and star forming regions, then only the line-of-sight component of the magnetic field, $B_{\|}$, can be determined. It can be shown that

$T_{V}(\nu)=-C \frac{\mathrm{d} T_{I}(\nu)}{\mathrm{d} \nu} B_{\|}[G]$,

where $T_{V}$ is the temperature of the Stokes- $V$ spectrum (the difference between the left- and right-circularly polarized spectra), $T_{I}$ is the temperature of the Stokes- $I$ spectrum (approximated as the sum of the left- and rightcircularly polarized spectra) and $\nu$ is the frequency. The term $C$ gives the left-right line frequency split with magnetic field strength $\left(\Delta \nu_{\mathrm{g}} / B\right)$ and is a constant for a given molecular transition; it has, for example, a value of $1.93 \mathrm{~Hz} / \mu \mathrm{G}$ for the $\mathrm{SO} J_{\mathrm{N}}=1_{2}-1_{1}$ transition (see Table 1 and Appendix A).

All processing of the spectral line data, including the production of the Stokes- $V$ and $I$ spectra, was done in the software environment CLASS ${ }^{1}$. The line-of-sight $B$ field $\left(B_{\|}\right)$was determined by scaling the $I$-derivative to the $V$ spectrum (Eq. (1)), by method of least-squares-fit.

Long-period waviness, attributable to receiver effects and the bandpass, was present in the baselines of all the

\footnotetext{
1 Developed by IRAM and the Observatoire de Grenoble.
}

$V$-spectra; it was removed by either of two methods. The baselines of some spectra were directly fitted with an $n$th order polynomial and then removed by subtracting the polynomial model. This procedure proved satisfactory for the narrow line sources (e.g. NGC 2071A, S140IR, the dark cloud cores), since their spectra contained ample baseline and since any potential Zeeman response within the confines of the narrow line would be distinct from the long-period waviness in the baseline. A different procedure, however, was needed for the spectra of the broadline sources (e.g. ORI-KL, Sgr B2N and G5.89), since in those cases the baselines are more limited in extent, thus making difficult their characterization and placing at risk the accidental subtraction of a real Zeeman response from the $V$-spectrum. Instead, separate observations of line-free or "sky" positions were used to model the baselines. The longest integrations of such were performed daily, during antenna servicing periods, with the antenna positioned toward the zenith. Additionally, measurements of nearby sky positions were periodically taken while observing some of the Zeeman line splitting candidates. The baselines of the sky-spectra were characterized with $n$th order polynomial fits. The source $V$-spectra were significantly flattened (typically to better than 2nd order curvature) upon subtraction of the sky spectra baseline model.

\section{Results}

The clear result of our initial $\mathrm{SO}\left(J_{\mathrm{N}}=1_{2}-1_{1}\right)$ survey is that emission from this transition is detected only towards regions of known high-density (within our sensitivity limit) - not unexpected in view of the transition's high critical density of $>10^{6-7} \mathrm{~cm}^{-3}$ (Green 1994). It is also observed in two molecular outflows, but this may be due to chemical enhancement in the molecular column density. The transition was not detected in Taurus-like dark cloud cores which, however, appeared strongly in our complementary CCS observations. Conversely, the CCS emission is weak or absent in warm star forming regions. The line parameters of the sources observed are presented in Table 2.

\subsection{Description of the Zeeman candidates}

In this section we briefly describe the sources selected for the Zeeman study, identifying and detailing the specific sub-regions believed coupled to the observations. We present, in Tables 3a and b, Cols. 2-6, a compilation of their physical characteristics (temperature, size, column density, number density, mass) gathered from the literature.

SO \& OH associated with dense star forming cores

$\rho \mathbf{O p h}-\mathbf{B}-$ The SO $J_{\mathrm{N}}=1_{2}-1_{1}$ emission follows closely the distribution of $\mathrm{H}_{2} \mathrm{CO}\left(J_{\mathrm{K}} 2_{11}-2_{12}\right) 2 \mathrm{~cm}$ emission (Martin-Pintado et al. 1983) and peaks towards the compact $\mathrm{H}_{2} \mathrm{CO}$ cores detected with the VLA (Wadiak et al. 1985). The VLA cores contain $\sim 50 \%$ of the single-dish 
Table 2. Parameters of sources selected for Zeeman integration

\begin{tabular}{|c|c|c|c|c|c|c|c|c|}
\hline Source & $\begin{array}{c}\text { RA } \\
(1950)\end{array}$ & $\begin{array}{c}\text { Dec } \\
(1950)\end{array}$ & $\begin{array}{l}t_{\text {int }} \\
{[\mathrm{h}]}\end{array}$ & $\begin{array}{c}\Delta V_{\mathrm{ch}} \\
{\left[\mathrm{km} \mathrm{s}^{-1}\right]}\end{array}$ & $\begin{array}{c}T_{1}(I) \\
{[\mathrm{K}]}\end{array}$ & $\begin{array}{l}V_{\mathrm{LSR}} \\
{\left[\mathrm{km} \mathrm{s}^{-1}\right]}\end{array}$ & $\begin{array}{l}\Delta V_{\text {line }} \\
{\left[\mathrm{km} \mathrm{s}^{-1}\right]}\end{array}$ & $\begin{array}{r}T(V) / T(I) \\
\left(10^{-3}\right)\end{array}$ \\
\hline \multicolumn{9}{|l|}{ SO Sources } \\
\hline W3-IRS4(S) & 02:21:44.0 & $61: 52: 32$ & 16.2 & 0.28 & 0.16 & -42.6 & 3.4 & 32.3 \\
\hline Orion-KL & $05: 32: 46.7$ & $-05: 24: 20$ & 4.3 & 1.12 & 0.93 & 9.0 & 23.5 & 8.7 \\
\hline NGC 2071A & $05: 44: 20.7$ & $00: 22: 20$ & 4.4 & 0.14 & 0.54 & 9.5 & 0.8 & 23.3 \\
\hline VLA 1623 & $16: 23: 20.2$ & $-24: 16: 04$ & 2.8 & 0.14 & 1.62 & 3.0 & 0.8 & 7.6 \\
\hline$\rho$ Oph-B & $16: 24: 08.9$ & $-24: 22: 41$ & 4.5 & 0.14 & 0.88 & 3.7 & 0.9 & 16.3 \\
\hline Sgr B2(N) & $17: 44: 10.1$ & $-28: 21: 17$ & 5.4 & 0.84 & 2.20 & 65.1 & 14.1 & 4.7 \\
\hline G5.89 & $17: 57: 26.8$ & $-24: 03: 56$ & 7.5 & 0.56 & -0.73 & 8.3 & 6.0 & 9.6 \\
\hline G10.62 & $18: 07: 30.7$ & $-19: 56: 29$ & 8.7 & 0.56 & $\begin{array}{r}-0.42 \\
0.04\end{array}$ & $\begin{array}{r}0.2 \\
-6.6\end{array}$ & $\begin{array}{l}3.0 \\
4.9\end{array}$ & 13.6 \\
\hline S140 SO & $22: 17: 42.0$ & 63:04:00 & 12.3 & 0.28 & 0.33 & -7.0 & 2.1 & 13.6 \\
\hline NGC 7538-IRS11 & $23: 11: 40.9$ & $61: 10: 48$ & 15.0 & 0.28 & 0.21 & -56.2 & 3.6 & 21.8 \\
\hline \multicolumn{9}{|l|}{ OH Sources } \\
\hline $\begin{array}{l}\text { G10.62 } \\
\text { DR21(OH) }\end{array}$ & $\begin{array}{l}18: 07: 30.7 \\
20: 37: 13.9\end{array}$ & $\begin{array}{r}-19: 56: 29 \\
42: 12: 11\end{array}$ & $\begin{array}{l}12.1 \\
19.7\end{array}$ & $\begin{array}{l}1.09 \\
1.09\end{array}$ & $\begin{array}{l}-0.28 \\
-0.31 \\
-0.09\end{array}$ & $\begin{array}{r}-2.0 \\
-5.1 \\
-10.3\end{array}$ & $\begin{array}{l}6.7 \\
4.9 \\
8.3\end{array}$ & $\begin{array}{l}9.1 \\
9.4\end{array}$ \\
\hline \multicolumn{9}{|l|}{ CCS Sources } \\
\hline L1498 & 04:07:55.8 & $25: 01: 33$ & 25 & 0.02 & 1.84 & 7.82 & 0.17 & 5.9 \\
\hline TMC-1C & 04:38:31.5 & $25: 55: 00$ & 28 & 0.02 & 1.83 & 5.37 & 0.20 & 5.8 \\
\hline TMC-1SE & 04:38:50.1 & $25: 32: 30$ & 23 & 0.02 & 2.19 & 5.66 & 0.14 & 4.7 \\
\hline
\end{tabular}

Note: The source names are contained in Col. 1 and the positions (in equatorial coordinates), in Cols. 2 and 3 , with the integration time in Col. 4. Column 5 gives the velocity width of the channels. Columns 6 through 8 give the line parameters, based on Gaussian fits to the spectrum. The line intensities, $T_{1}$, are presented as main beam brightness temperatures. The $1 \sigma$ rms noise of the Stokes $V$-spectrum, normalized to the peak total power $I$-profile, are given in Col. 9

flux. The $\mathrm{H}_{2} \mathrm{CO}$ ridge is $3.2 \times 0.7^{\prime}$ in size and the $\mathrm{SO}$ spectra throughout this region share a singular velocity $\left(V=3.6 \mathrm{~km} \mathrm{~s}^{-1}\right)$ and linewidth $\left(\Delta V=1.1 \mathrm{~km} \mathrm{~s}^{-1}\right)$ identical to that of $\mathrm{NH}_{3}$ line emission observed (Martin-Pintado et al. 1983). Densities in excess of $10^{6} \mathrm{~cm}^{-3}$ are implied by the detection of the $2 \mathrm{~cm} \mathrm{H}_{2} \mathrm{CO}$ line in emission.

Sgr B2 (N) - The complex structure of the Sgr-B2 star forming cloud has been characterized as having three distinct components (see e.g. Hüttemeister et al. 1995, and Ref. therein): embedded in a dense cloud of size $1 \times 2^{\prime}\left(n \sim 10^{5} \mathrm{~cm}^{-3}, N \sim 10^{24} \mathrm{~cm}^{-2}, T \sim 100 \mathrm{~K}\right)$ there are three prominent compact ultra-dense clumps, referred to as $\mathrm{N}$ (orth), $\mathrm{M}(\mathrm{id})$ and $\mathrm{S}$ (outh), with volume densities $n \geq 10^{7} \mathrm{~cm}^{-3}$ and column densities as high as $10^{25} \mathrm{~cm}^{-2}$. The bulk of the mass, however, is contained in an extended envelope of somewhat lower density $\left(10^{3} \mathrm{~cm}^{-2}\right)$. Kinematically, it is difficult to discriminate between the dense ridge and the embedded cores in Sgr B2 $(\mathrm{N})$, both sharing bulk velocities of $V_{\mathrm{lsr}} \sim 65 \mathrm{~km} \mathrm{~s}^{-1}$ and linewidths of $\sim 15 \mathrm{~km} \mathrm{~s}^{-1}$. The higher velocity component at $81 \mathrm{~km} \mathrm{~s}^{-1}$ seen in our SO $J_{\mathrm{N}}=1_{2}-1_{1}$ spectrum likely arises from the envelope (Hüttemeister et al. 1995). The SO emission does not show much of variation between the $\mathrm{N}$ and $\mathrm{S}$ position, therefore we tentatively associate the SO gas with the dense ridge. Any contribution from the ultra-dense cores is difficult to judge with our resolution.
G5.89 (W28 A2) - A bright ultra-compact H II region, whose exciting source (equivalent to a O5 ZAMS object) must be very young and still deeply embedded in its high density parental cloud $\left(n_{\mathrm{H}_{2}}=10^{7}-10^{8} \mathrm{~cm}^{-3}\right.$ along its inner edge; Lightfoot et al. 1984). Associated $\mathrm{NH}_{3}$ emission is confined to within a $5^{\prime \prime}$ radius region centered on the main continuum component (Gómez et al. 1991) and is seen in absorption at velocities of $V=7 \mathrm{~km} \mathrm{~s}^{-1}$, similar to that of the observed SO $J_{\mathrm{N}}=1_{2}-1_{1}$ line. Virial densities compare nicely with the bulk densities derived from $\mathrm{C}^{34} \mathrm{~S}$ excitation studies (Cesaroni et al. 1991, 1992).

G10.62 - Our position is centered on the brightest continuum source in the G10.62 star forming region. $\mathrm{NH}_{3}$ absorption, as well as the kinematics of associated gas, imply that the HII region is surrounded by a rotating and infalling gaseous core/envelope structure (Ho \& Haschick 1986; Keto et al. 1987). The SO has the same redshifted velocity as $\mathrm{NH}_{3}$ and $\mathrm{H}_{2} \mathrm{CO}$ absorption lines $\left(V=0 \mathrm{~km} \mathrm{~s}^{-1}\right)$, identifying this component as the dense infalling material of the surrounding "core" foreground to the H II region. The weak SO emission corresponds kinematically to the dense core previously observed with the optically thin and high-gas density sampling $\mathrm{C}^{18} \mathrm{O}$ (Ho et al. 1994) and $\mathrm{C}^{34} \mathrm{~S}$ lines (Hauschildt et al. 1993).

W3-IRS4 - Our position is located $\sim 20^{\prime \prime}$ south of IRS 4 (the second brightest infrared source in the W3 star forming complex; Wynn-Williams et al. 1972) and corresponds 
Table 3a. Source physical parameters (OH and SO observations)

\begin{tabular}{|c|c|c|c|c|c|c|c|c|c|c|c|}
\hline Source & & $\begin{array}{l}T_{\mathrm{K}} \\
{[\mathrm{K}]}\end{array}$ & $\begin{array}{c}<\text { size }> \\
{[\mathrm{pc}]}\end{array}$ & $\begin{array}{l}n\left(\mathrm{H}_{2}\right) \\
{\left[\mathrm{cm}^{-3}\right]}\end{array}$ & $\begin{array}{c}N\left(\mathrm{H}_{2}\right) \\
{\left[\mathrm{cm}^{-2}\right]}\end{array}$ & $\begin{array}{l}\text { Mass } \\
{\left[M_{\odot}\right]}\end{array}$ & Ref. & $\begin{array}{l}B_{\|}^{\mathrm{rms}} \\
{[\mathrm{mG}]}\end{array}$ & $\begin{array}{l}B_{\text {crit }} \\
{[\mathrm{mG}]}\end{array}$ & $\begin{array}{c}B_{\text {alf }} \\
{[\mathrm{mG}]}\end{array}$ & $\begin{array}{l}B_{\text {vir }} \\
{[\mathrm{mG}]}\end{array}$ \\
\hline \multicolumn{12}{|l|}{$\underline{\text { SO Sources }}$} \\
\hline W3-IRS4(S) & (HCN core) & 55 & 0.2 & $8(5)$ & $2-4(23)$ & $\sim 200$ & $19-22$ & 0.8 & $2-3$ & 1.5 & 1.4 \\
\hline Orion-KL & (doughnut) & 200 & $(0.02)$ & $>1(7)$ & few $(23)$ & 5 & $1-4$ & 1.5 & 0.8 & $\cdots$ & $\cdots$ \\
\hline \multirow[t]{2}{*}{$\rho$ Oph-B } & (halo) & 15 & 0.075 & $>1(6)$ & $2(23)$ & 6 & 11,12 & 0.2 & 1.6 & $>0.5$ & 0.3 \\
\hline & (compact core) & $\ldots$ & 0.03 & $4(6)$ & $4(23)$ & 4 & $\cdots$ & $\cdots$ & 3.2 & 0.9 & 0.7 \\
\hline \multirow[t]{2}{*}{ Sgr B2(N) } & (dense ridge) & 100 & 3.5 & $1(5)$ & $1(24)$ & $\ldots$ & 8,10 & 0.6 & 8 & $<2.3$ & 1.4 \\
\hline & (compact core) & 150 & 0.16 & $2(7)$ & $1(25)$ & $2(3)$ & $\cdots$ & $\ldots$ & 80 & $<32$ & 31 \\
\hline G5.89 & & 90 & 0.3 & $\sim 2(6)$ & $4-6(23)$ & $4-800$ & $16-18$ & 0.6 & $3-5$ & 4.3 & 3.0 \\
\hline G10.62 & & 100 & 0.1 & $2-4(6)$ & $2(24)$ & 1100 & $13-15$ & 0.5 & 16 & $2.1-3.1$ & 2.2 \\
\hline S140 SO & (SMM2) & 35 & 0.04 & $>4(6)$ & $>5(23)$ & 6.5 & $23-25$ & 0.4 & $>4$ & $>2.1$ & 2.8 \\
\hline NGC 7538 & (20" E of IRS11) & $50^{*}$ & 0.20 & $6(5)$ & $4(23)$ & 250 & $5-7$ & 0.7 & 3.2 & 1.4 & 1.6 \\
\hline NGC 2071A & (outflow) & $\ldots$ & $\ldots$ & $\ldots$ & $\ldots$ & $\ldots$ & $\ldots$ & 0.25 & $\ldots$ & $\ldots$ & $\ldots$ \\
\hline VLA 1623 & (outflow) & $\ldots$ & $\ldots$ & $\ldots$ & $\ldots$ & $\ldots$ & $\cdots$ & 0.1 & $\ldots$ & $\ldots$ & $\ldots$ \\
\hline \multicolumn{12}{|l|}{$\underline{\text { OH Sources }}$} \\
\hline G10.62 & & 100 & 0.1 & $2-4(6)$ & $2(24)$ & 1100 & $13-15$ & 1.2 & 16 & $2.1-3.1$ & 2.2 \\
\hline $\mathrm{DR} 21(\mathrm{OH})$ & (MM1) & $>80$ & $<0.1$ & $2-4(7)$ & $4(24)$ & $2-300$ & $26-29$ & 2.0 & 32 & $11-16$ & $>6$ \\
\hline
\end{tabular}

(1) Plambeck et al. (82), (2) Stutzki et al. (88), (3) Wright et al. (96), (4) Blake et al. (87), (5) Minchin et al. (94), (6) Sandell (priv. comm.), (7) Zylka (priv. comm.), (8) Hüttemeister et al. (93,95) \& ref. therein (10) Vogel et al. (87), (11) Martin-Pintado et al. (83), (12) Wadiak et al. (85) (13) Keto et al. (87) (14) Hauschildt et al. (93), (15) Ho et al. (94), (16) Gomez et al. (91), (17) Cesaroni et al. (91), (18) Cesaroni et al. (92), (19) Helmich et al. (94), (20) Wright et al. (84), (21) Tieftrunk et al. (95), (22) Oldham et al. (94), (23) Ungerechts et al. (86), (24) Zhou et al. (94), (25) Minchin et al. (95), (26) Mangum et al. (91), (27) Mangum et al. (92), (28) Padin et al. (89), (29) Jones et al. (94).

Table 3b. Source physical parameters (CCS observations)

\begin{tabular}{|c|c|c|c|c|c|c|c|c|c|c|c|c|}
\hline Source & $\begin{array}{l}T_{K} \\
{[\mathrm{~K}]} \\
\end{array}$ & $\begin{array}{c}<\text { size }> \\
{[\mathrm{pc}]}\end{array}$ & $\begin{array}{l}n\left(\mathrm{H}_{2}\right) \\
{\left[\mathrm{cm}^{-3}\right]}\end{array}$ & $\begin{array}{l}\text { Mass } \\
{\left[M_{\odot}\right]}\end{array}$ & $\begin{array}{c}M_{\mathrm{J}} \\
{\left[M_{\odot}\right]} \\
\end{array}$ & $\begin{array}{c}\sigma_{\left(\mathrm{H}_{2}\right)} \\
{\left[\mathrm{km} \mathrm{s}^{-1}\right]}\end{array}$ & $\begin{array}{c}\sigma_{\mathrm{nt}} \\
{\left[\mathrm{km} \mathrm{s}^{-1}\right]}\end{array}$ & $\begin{array}{l}B_{\|}^{\mathrm{rms}} \\
{[\mu \mathrm{G}]}\end{array}$ & $\begin{array}{l}B_{\text {alf }} \\
{[\mu \mathrm{G}]}\end{array}$ & $\left(\frac{2 \mathcal{T}}{\mathcal{W}}\right)$ & $\left(\frac{2 \mathcal{T}}{\mathcal{M}_{\text {alf }}}\right)$ & $\left(\frac{2 \mathcal{T}}{\mathcal{M}_{\text {obs }}}\right)$ \\
\hline \multicolumn{13}{|l|}{$\underline{\mathrm{CCS}}$} \\
\hline L1498 & 9.7 & 0.07 & $8(4)$ & $\sim 0.6$ & 0.8 & 0.20 & 0.040 & 70 & 13 & 1.7 & 92 & $>1.1$ \\
\hline TMC-1C & 9.0 & 0.11 & $2-6(4)$ & $0.6-1.9$ & $1.4-0.8$ & 0.19 & 0.055 & 78 & $9-16$ & $0.8-2.6$ & 42 & $0.2-0.6$ \\
\hline TMC-1SE & 8.9 & $\ldots$ & $>4(4)$ & $\ldots$ & $<1.0$ & 0.19 & 0.043 & 51 & $>10$ & $\ldots$ & 70 & $>0.9$ \\
\hline
\end{tabular}

Observables are taken from, (1) Fiebig (90), (2) Cox et al. (89), (3) Cox (priv. comm.).

Note: For the calculation of $M_{\mathrm{obs}}$ we have assumed $B^{2}=3 B_{\|}^{2}$, a statistical approach that may not apply in this particular case.

to the dense core identified by other molecular tracers (HCN, Wright et al. 1984; $\mathrm{C}^{18} \mathrm{O}$, Oldham et al. 1994; $\mathrm{C}^{34} \mathrm{~S}$, Tieftrunk et al. 1995) as well as with one of two maxima in $800 \mu \mathrm{m}$ continuum emission (Richardson et al. 1989). The velocity and linewidth of the $\mathrm{SO}$ emission are in agreement with those of $\mathrm{C}^{18} \mathrm{O}$ measured by Tieftrunk et al. (1995). The nonisotopic CO lines, in contrast, are nonsymmetric, double peaked, and display larger linewidths ranging between $\Delta V=9$ and $18 \mathrm{~km} \mathrm{~s}^{-1}$ (Hasegawa et al. 1994; Dickel et al. 1980).

Orion-KL - Following up on the detection of SO emission by Gottlieb et al. (1978), the interferometric studies by Plambeck et al. (1982) and Wright et al. (1996) indicated that $\mathrm{SO} J_{\mathrm{K}}=2_{2}-1_{1}$ emission is localized to within a $11^{\prime \prime} \times 19^{\prime \prime}$ region centered on the IRC2 source, known now as the low-velocity part of the "plateau" feature. Our measurements are consistent with these findings; the SO $J_{\mathrm{N}}=1_{2}-1_{1}$ line parameters are similar to those determined by Gottlieb et al., and substantial emission is recorded only within a single beam centered near the IRC2 source. Plambeck et al. (1982) hypothesize that the SO emission arises from an expanding torus which surrounds and perhaps collimates a bipolar outflow arising from IRC2. 
S 140 SO - S 140 is a crescent shaped H II region bordering the edge of the dark cloud L1204. The SO $J_{\mathrm{N}}=1_{2}-1_{1}$ emission is centered $\sim 15^{\prime \prime}$ north-east of S 140-IRS1, the location of strongest $\mathrm{C}^{(34)} \mathrm{S}$ (Zhou et al. 1994) and $\mathrm{NH}_{3}$ (Ungerechts et al. 1986) emission. The SO peak does, however, coincide with SMM2, a dense compact core identified by its dust continuum emission (Minchin et al. 1995), but not observed in previous molecular line studies.

NGC 7538 IRS11 - The only position in the NGC 7538 star forming cloud where appreciable $\mathrm{SO} J_{\mathrm{N}}=1_{2}-1_{1}$ emission was detected is offset $20^{\prime \prime}$ east of the embedded IRS11 source. Towards this position a dense dust core has been detected (see reference in Minchin et al. 1994). The physical parameters for this compact core, given in Table 3a, are deduced from unpublished dust continuum observations (Sandell \& Zylka, priv. comm.), assuming $T=50 \mathrm{~K}$.

DR21 (OH) - This position, also referred to as W75S (Wilson \& Mauersberger 1990) and DR21(OH)M, is located $\sim 3^{\prime}$ north of the DR21 H II region toward the site of a prominent maser and far IR maximum. DR21 $(\mathrm{OH})$ is toward the strongest of four CS peaks, separated by 30-50", in the DR21 (OH) region (Chandler et al. 1993). Judging from the velocity of the $\mathrm{OH}$ emission, it arises from the dust core MM1 (Padin et al. 1989).

An exploratory SO observation was also made toward W3 $(\mathbf{O H})$ where, in an earlier $\mathrm{OH}$ Zeeman absorption line study, Güsten et al. (1994) found a $3 \mathrm{mG}$ line-ofsight field within its dense gas at $V=-45 \mathrm{~km} \mathrm{~s}^{-1}$. We indeed detected $\mathrm{SO}$ toward $\mathrm{W} 3(\mathrm{OH})$, but only in emission, and at a velocity $\left(V=-47 \mathrm{~km} \mathrm{~s}^{-1}\right)$ similar to that of a second $\mathrm{OH}$ absorption component observed (in the earlier study) which gave no indication of a magnetic field above a limit of $\sim 0.4 \mathrm{mG}$. We therefore did not integrate further in the attempt to make an SO Zeeman measurement toward $\mathrm{W} 3(\mathrm{OH})$. The $-47 \mathrm{~km} \mathrm{~s}^{-1}$ component of gas is apparently distributed in a relatively extended envelope $\left(\sim 2^{\prime}\right)$ around the H II region (Wilson et al. 1991; Dickel \& Goss 1987), whereas the $-45 \mathrm{~km} \mathrm{~s}^{-1}$ component containing the strong $B$ field is of somewhat higher density $\left(10^{6.8} \mathrm{~cm}^{-3}\right)$ and more closely borders the $\mathrm{H}$ II region along its western edge (Wink et al. 1994; Reid et al. 1987; Guilloteau et al. 1984). So, unfortunately, no independent confirmation of the earlier $\mathrm{OH}$ results has been possible.

\section{$\underline{\text { SO associated with molecular outflows. }}$}

NGC 2071A - We have centered on an SO emission peak, mapped by Schmid-Burgk \& Muders (1994), located $\sim 3^{\prime}$ northwest of the NGC 2071 bipolar outflow (Bally 1982; Snell et al. 1984; Scoville et al. 1986; MoriartySchieven et al. 1989). Both the $J_{\mathrm{N}}=1_{0}-0_{1}$ emission lines observed by Schmid-Burgk \& Muders and the $J_{\mathrm{N}}=1_{2}-1_{1}$ emission lines observed in this study are relatively narrow $\left(\Delta V=0.9 \mathrm{~km} \mathrm{~s}^{-1}\right)$, in contrast to high excitation SO $J_{\mathrm{N}}=6_{5}-5_{4}$ emission observed by Chernin \& Masson (1993) which have complex line structure and large velocity width and are correlated to the ends of the high velocity CO outflows (Chernin \& Masson 1992). The CS emission is distributed symmetrically, within a 0.5 region, on the cluster of infrared sources (IRS 1, 2, and 3; Persson et al. 1981) central to the outflow activity (Zhou et al. 1991). Our position (NGC 2071A) is outside of the region mapped previously by molecular line studies. We detect no SO line emission towards a second position, NGC 2071, 22" south of IRS 1.

VLA 1623 - A compact VLA continuum source and class 0 protostellar candidate centered on well-collimated molecular outflow, the only such flow found in the $\rho$-Oph starforming complex to date (André et al. 1993; André et al. 1990). Our position is toward the tip of the $2^{\prime}$-scale redshifted molecular lobe. Indeed, the SO $J_{\mathrm{N}}=1_{2}-1_{1}$ spectrum appears to be slightly asymmetric, perhaps possessing a modest redward tail. The $F W H M$ of the SO line is $0.8 \mathrm{~km} \mathrm{~s}^{-1}$. By comparison, the ${ }^{12} \mathrm{CO}$ spectra (André et al. 1990) associated with the molecular flow have velocity widths on the order of $\Delta V=\sim 8 \mathrm{~km} \mathrm{~s}^{-1}$ and are likely blends of several velocity components. Also coinciding with our position is the bright infrared source GSS 30 (Graasdalen et al. 1973).

\section{$\underline{\text { CCS towards dark cloud cores }}$}

From our survey of dark cloud cores in the Orion and Taurus region we selected three dark cores for deep Zeeman integrations: L1498, TMC-1C, and TMC-1SE. To identify the best suited positions (strongest lines with cleanest possible Gaussian profiles) the cores were first mapped in the $\operatorname{CCS}\left(J_{\mathrm{N}}=2_{1}-1_{0}\right)$ transition at $22.34 \mathrm{GHz}$ (42" angular resolution). Their kinetic temperatures were determined using observations of their lower $\mathrm{NH}_{3}$ rotational transitions (Fiebig 1990) and their $\mathrm{H}_{2}$ densities were estimated from a multi-transition study of $\mathrm{C}_{3} \mathrm{H}_{2}$ (Cox et al. 1989; Cox, priv. comm.) (Table 3b, Col. 4).

\subsection{B field determinations}

We detect no line-of-sight magnetic field $\left(B_{\|}\right)$above the $3 \sigma$ uncertainty level toward any of the sources observed, either by their SO $J_{\mathrm{N}}=1_{2}-1_{1} \quad \mathrm{OH}^{2} \Pi_{3 / 2} \quad J=7 / 2$ or CCS line emission/absorption. Figures $1 \mathrm{a}-\mathrm{c}$ display the Stokes- $V$ and Stokes- $I$ spectra for the SO line. With exception only to $\operatorname{SgrB} 2(\mathrm{~N})$, no large-scale deviations in the $V$-spectra are seen within the velocity limits of their respective lines. The $\mathrm{SgrB} 2(\mathrm{~N})$ displays a signature in its $V$-spectrum consistent with a field at the $1 \sigma$ level $\left(B_{\|}=0.57 \mathrm{mG}\right)$. The broadness and complex shape of the line, however, makes its interpretation difficult at this level.

In Table 2, Col. 9 , we present the $1 \sigma$ limits on $V / I$. In Table 3a (Col. 8) and 3b (Col. 9), the corresponding $1 \sigma$ limit on the magnetic field strength $B_{\|}$for each region is given. 

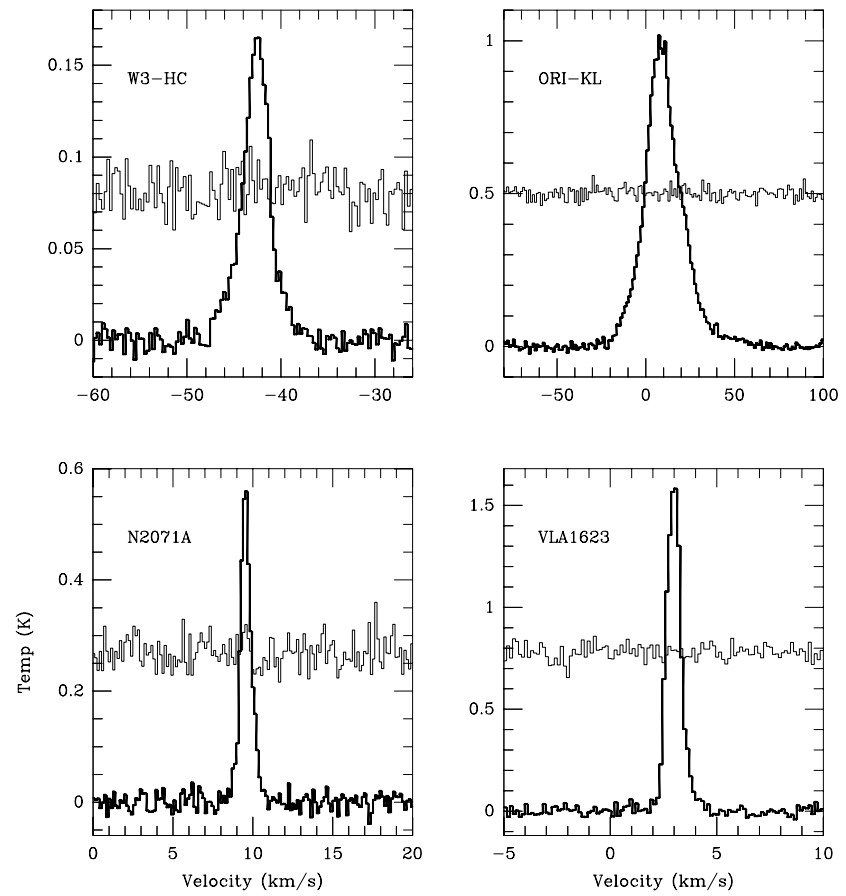

Fig. 1. The SO $J_{\mathrm{N}}=1_{2}-1_{1}$ Stokes- $V$ difference spectra (thin solid line) and the Stokes- $I$ total power spectra (thick line) toward the sources indicated in upper left corner of each panel. Note that there is no sign in any of the Stokes- $V$ spectra of a Zeeman response above the level of the noise

\section{Discussion}

\subsection{Comparison to theoretical estimates of the $B$ field}

\section{$\underline{\mathrm{SO} \text { and } \mathrm{OH} \text { results }}$}

Several basic formulations have been developed to estimate magnetic field strengths in clouds. All are based on meaningful physical footings, but approach the problem differently in the way assumptions are made about the equipartition of energies and source geometries. The various formulations require different "observables" $\left(\left[N_{\mathrm{H}_{2}}\right]\right.$, $\left[n_{\mathrm{H}_{2}}, \Delta V\right]$, and $\left.[\Delta V, R]\right)$, some obtained by independent means. It is thus worthwhile to review all three theoretical methods and to compare the range of estimates they produce to the measured field limits.

In the absence of other large-scale supportive mechanisms (such as turbulence), the critical magnetic field needed to support a self-gravitating cloud against collapse is,

$B_{\text {crit }}=810^{-21} N_{\mathrm{H}_{2}}\left(\mathrm{~cm}^{-2}\right) \quad \mu \mathrm{G}$;

this based on the derivation by Mouschovias \& Spitzer (1976), where the expressions were tuned to the results of exact numerical models of initially uniform, spherical magnetic clouds, and using a mean molecular mass of $\mu=$ $2.33 m_{\mathrm{H}}$.

A second means of estimating the magnetic field in a cloud is by its "nonthermal" velocity dispersion. There have been a number of mechanisms suggested to explain the nonthermal component, or that in excess of the thermal contribution to the linewidth, often observed in lines
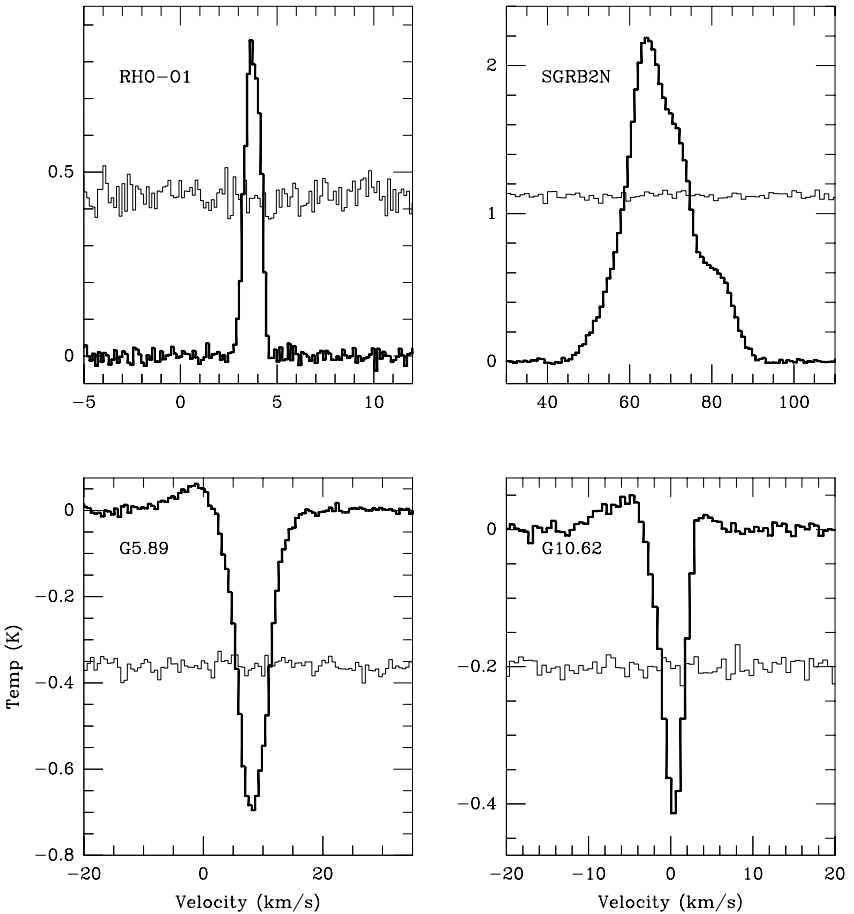

Fig. 2. The SO $J_{\mathrm{N}}=1_{2}-1_{1}$ Stokes- $V$ difference spectra (thin solid line) and the Stokes- $I$ total power spectra (thick line) toward the sources indicated in upper left corner of each panel. Note that, with exception of Sgr B2 (N) (which is marginal), there is no sign in any of the Stokes- $V$ spectra of a Zeeman response above the level of the noise
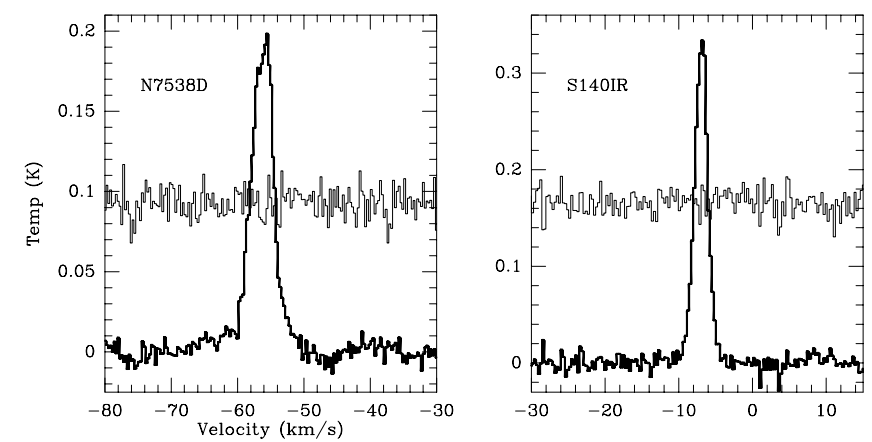

Fig. 3. The SO $J_{\mathrm{N}}=1_{2}-1_{1}$ Stokes- $V$ difference spectra (thin solid line) and the Stokes- $I$ total power spectra (thick line) toward the sources indicated in upper left corner of each panel. Note that there is no sign in any of the Stokes- $V$ spectra of a Zeeman response above the level of the noise

toward molecular clouds. Stellar winds or outflows, were among the first to be cited (Norman \& Silk 1980; Larson 1981; Zuckerman \& Evans 1974). Upon further consideration, however, such inputs are now believed to be relatively ineffective since the supersonic turbulence they produce should be quickly dissipated in shocks. Instead, long-wavelength and large-amplitude MHD waves within the cloud are thought to better fit the role, since these waves are long-lived so long as their propagation speed is below the local Alfven velocity. Thus, a means of estimating the upper limit to the $B$ field is to compare the 
nonthermal contribution of a cloud's velocity dispersion, $\sigma_{\text {nt }}$, to its Alfven velocity, $V_{\text {alf }}=B /\left(4 \pi \mu n_{\mathrm{H}_{2}}\right)^{1 / 2}$, where $n_{\mathrm{H}_{2}}$ is the volume density. Following Myers \& Goodman (1988), we adopt $\sigma_{\mathrm{nt}}=\sqrt{3} \cdot V_{\text {alf }}$, leading to $\left(\mu=2.33 m_{\mathrm{H}}\right)$

$B_{\text {alf }}=1.2 n_{\mathrm{H}_{2}}^{0.5}\left(\mathrm{~cm}^{-3}\right) \sigma_{\mathrm{NT}}\left(\mathrm{km} \mathrm{s}^{-1}\right) \quad \mu \mathrm{G}$.

The nonthermal velocity dispersion is taken to be the difference, in quadrature, between the observed velocity dispersion and the thermal component

$\sigma_{\mathrm{nt}}^{2}=\sigma_{\mathrm{obs}}^{2}+\sigma_{\mathrm{th}}^{2}=\frac{\Delta V_{\mathrm{obs}}^{2}}{8 \ln 2}-\frac{k T}{\mu_{\mathrm{obs}}}$,

where $\Delta V_{\text {obs }}$ is the FWHM linewidth of the observed molecule, $\mu_{\mathrm{obs}}$ is the molecule's mass, and $T$ is the kinetic temperature of the gas. For the case of the SO emission lines, the thermal contributions to the FWHM linewidths are small $\left(\Delta V_{\mathrm{th}}=0.03 T^{0.5}\right)$ due to the relatively large mass of this molecule and the nonthermal component dominates in all sources; for the narrow CCS lines the correction is significant.

Another means of estimating the magnetic field in a spherical cloud with negligible thermal support is by assuming the conditions of both magnetic and virial equilibrium in the cloud. The resulting relation is,

$B_{\mathrm{vir}}=15 \alpha^{-1} \Delta V^{2} R^{-1} \mu \mathrm{G}$,

where $R$ is the radius of the cloud in parsecs, $\Delta V$ the (nonthermal) $F W H M$ of the line in $\mathrm{km} \mathrm{s}^{-1}$, and $\alpha$ a scaling factor (ranging somewhere between 1.1 and 1.3; Myers \& Goodman 1988; McKee et al. 1993).

In Cols. 9-11 of Table 3a, we provide the critical magnetic field estimates, determined using the three previously discussed methods, for the twelve $\mathrm{SO} / \mathrm{OH}$ sources observed in this study using the "observables" from Cols. 2-5 $\left(T_{\mathrm{K}}, d(\right.$ size $), n_{\mathrm{H}_{2}}$, and $\left.N_{\mathrm{H}_{2}}\right)$. The critical field estimates $B_{\text {crit }}, B_{\text {alf }}$, and $B_{\text {vir }}$ for any given source vary by as much as a factor of a few with G10.62 being the extreme case with a $B_{\text {crit }}$ and $B_{\text {vir }}$ that differ by a factor of seven. While the $B_{\text {crit }}$ formulation generally gives the largest critical field results (Table 3a, Col. 9) of the three formulations, it is the most straightforward requiring the fewest assumptions on equipartition and source geometry and requiring only an estimate of the source column density $\left(N_{\mathrm{H}_{2}}\right)$, the most reliable of our observed parameters. We therefore adopt the $B_{\text {crit }}$ values for comparison to our observational $B_{\|}$field limits and for the discussion to follow.

In all but one (Orion-KL) of the eight SO sources for which we were able to calculate the $B_{\text {crit }}$ field values (Table 3a, Col. 9) the observationally determined $3 \sigma B_{\|}$ field limits (three times the value of Col. 8 in Table 3a) fall well below their corresponding $B_{\text {crit }}$ values. Even if our Sgr B2(N) data does indicate a $B_{\|}$field of $1.2 \mathrm{mG}$, which we very much question (Sect. 4.2), it is still well below the $B_{\text {crit }}$ of $\sim 8 \mathrm{mG}$ of estimated for this region. Moreover, while we conservatively use $3 \sigma$ values as the upper limit to the $B_{\|}$field in these regions, we are, in fact, confident that the $V$-spectra show no magnetic field signatures down even at the $1 \sigma$ level. If we were to go by the less stringent $1 \sigma$ criteria, then in no instance would the observed $B_{\|}$magnetic field limits exceed the critical $B$ field determinations for the sources, whether it is that of $B_{\text {crit }}, B_{\text {alf }}$ or $B_{\text {vir }}$.

The $3 \sigma$ observed limits for the $\mathrm{H}$ II regions G10.62 and $\operatorname{DR} 21(\mathrm{OH})\left(B_{\|}=3.6\right.$ and $6.0 \mathrm{mG}$, respectively), determined from the $\mathrm{OH}^{2} \Pi_{3 / 2} \quad J=7 / 2$ observations, also fall far short of their $B_{\text {crit }}$ values, implying supercriticality in these clouds. Furthermore, the observations indicate no fields comparable to that of $\mathrm{W} 3(\mathrm{OH})$, found in our earlier study. The SO observations of G5.89 and G10.62 provide even tighter constraints on the fields in $\mathrm{H}$ II regions, with $3 \sigma B_{\|}$limits averaging $1.6 \mathrm{mG}$. Given the results of this survey, the W3 $(\mathrm{OH})$ region - earlier reported to have a line-of-sight $B$ field of $\sim 3 \mathrm{mG}$ in its dense thermal gas (Güsten et al. 1994) - appears to be the exception, rather than the norm, in terms of its magnetic field.

The outflow sources NGC 2071A and VLA 1623 are the few of its kind to have been observed in Zeeman studies. Their observations also provide among the lowest field limits $\left(B_{\|}(3 \sigma)=0.3\right.$ and $\left.0.75 \mathrm{mG}\right)$ in our study. Unfortunately, we have little physical information on the NGC 2071A and VLA 1623 regions - either from our observations or the literature - and, therefore, no means of determining any of the critical $B$ field values $\left(B_{\text {crit }}\right.$, $B_{\text {alf }}$ or $B_{\text {vir }}$ ) by any of the methods. While the SO lines measured toward the other eight sources consistently agree with those of other high density tracers, implying that this transition traces hugh volume and column densities, according to chemical/shock models (Neufeld \& Dalgarno 1989; Mitchell 1984; Harquist et al. 1980) and some observations (Martin-Pintado et al. 1992), compounds such as $\mathrm{SO}$ and $\mathrm{SiO}$ may also be strongly enhanced in post shock environments. This is likely the situation toward VLA 1623 and NGC 2071A where the $\mathrm{SO}$ emision is enhanced along the edge of the outflow. The critical field determinations of these regions and their comparison to our measured field values is thus deferred until the source parameters are better known.

\section{$\underline{\text { CCS results }}$}

In those dark cloud cores observed by their CCS transition, the thermal linewidth components correspond to the kinetic temperatures derived from the $\mathrm{NH}_{3}$ excitation studies (Fiebig 1990), suggesting that thermal support plays a major role in the stability of these cores. In such case, the relations for $B_{\text {crit }}$ and $B_{\text {vir }}$ discussed previously (Eqs. (2) and (5), respectively) do not apply. We therefore take a somewhat different approach from above in estimating and comparing to the critical field limits of these clouds.

In Table $3 \mathrm{~b}$ we characterize the dense cores by their virial expressions for the gravitational energy $\mathcal{W}=(3 / 5) a G M^{2} / R$, the kinetic energy $\mathcal{T}=(3 / 2) M \sigma_{\mathrm{H}_{2}}^{2}$, 
and the magnetic energy $\mathcal{M}=(1 / 3) b B^{2} R^{3} \quad$ (e.g. Crutcher 1999, with $a=1.2$ and $b=0.3$ following Mckee et al. 1993). The physical parameters of the clouds are derived from $\mathrm{NH}_{3}$ (Fiebig 1990) and $\mathrm{C}_{3} \mathrm{H}_{2}$ studies (Cox et al. 1989; Cox, priv. comm.). The Jeans, $M_{\mathrm{J}}$, is presented in Col. 6 and the thermal $\left(\sigma_{\left(\mathrm{H}_{2}\right)}\right)$ and nonthermal linewidths $\left(\sigma_{\mathrm{nt}}\right)$ in Cols. 7 and 8 , respectively.

Within the uncertainties, the mass of the cores compare to their Jeans mass, the ratio of the kinetic to gravitational energy $2 \mathcal{T} / \mathcal{W}$ is $\sim 1$, suggesting that the cores are evolving in approximate equilibrium. If we assume the non-thermal velocity dispersion, $\sigma_{\mathrm{nt}}$ (Col. 8), provides some measure of the turbulent field component, $B_{\text {alf }}$, then $2 \mathcal{T} / \mathcal{M}_{\text {alf }} \sim 42-92$ (Col. 12). Our observational limits to the large scale field strength, $B_{\|}$, as sensitive as they are, do not allow a critical assessment of any large scale field on the energetics of the core since $2 \mathcal{T} / \mathcal{M}_{\text {obs }} \sim 1$ (Col. 13) (assuming $B^{2}=3^{\star} B_{\text {par }}$ - a statistical approach that may not apply to the small number of cores observed), i.e., $B_{\|}$ exceeds $B_{\text {alf }}$ by a factor of $\sim 6$.

\section{The large scale magnetic field}

The $B_{\|}$upper limits found in this study, most being well below the critical $B$ field values determined, tend to support the assertion that the magnetic fields in these dense clouds are supercritical (not capable of supporting the cloud). However, as with all Zeeman studies we give the cautionary notice that the sample is limited and there are a number of factors involved with Zeeman measurements that can cause the underestimation of the actual $B$ field strength or avoid detection entirely, such as, (1) the alignment of an ordered magnetic field component out of the line of sight of the observer, and (2) substantial tangling and sign reversals of the field within the beam or region sampled by the beam. Nevertheless, we do not believe that in this study these effects significantly alter our results or conclusions.

Because Zeeman line measurements measure the component of the $B$ field parallel to the line of sight $B_{\|}=$ $B \cdot \cos (\theta)$, one can only infer the total field strength statistically and there is the risk of missing an ordered field, even a strong one, if oriented sufficiently close to within the plane of the sky. The total field $(B)$, or total field limit, is expected to be twice as large on average for a Zeeman derived sample of randomly oriented fields. It is, however, highly unlikely in a sufficiently large sample that all the fields observed are fortuitously aligined such that they avoid detection entirely. Adopting the statistical methods of Crutcher et al. (1993), we find that the probability of not detecting any field above the $3 \sigma$ levels obtained, assuming total field values $\left(B_{\text {tot }}\right)$ at the expected $B_{\text {crit }}$ levels, is only $0.04 \%$.

Another concern is if the field has complex structure or numerous sign reversals within the beam, leading to a diluted average of its actual value or a nondetection. Beam averaging is especially a consideration in HI Zeeman studies where the beamsizes are typically very large and the diffuse gas that is being probed is extended, often filling the entire beam. In this study, however, the CS and $\mathrm{NH}_{3}$ clump sizes (Table 3a, Col. 3), which we take as roughly representative of the SO clump sizes we observe, are far smaller than the antenna beam $F W H M$ of $63^{\prime \prime}$. We thus avoid averaging over large spatial areas (few pcs), but only on the scales of the $\mathrm{H}$ II regions, dense cloud cores, or accretion disks themselves (all sub-parsec except for Sgr $\mathrm{B} 2(\mathrm{~N}))$.

Unfortunately, very little is known regarding the typical coherence scale of magnetic fields on the level of dense molecular clouds and their dense sub-cores. Submillimeter and far infrared polarization studies, which infer the field direction by the alignment of grains, are one of the few means of detailing the fields on the scales of protostars (Holland et al. 1995) and dense condensations. Typical polarization studies have beamsizes of the order $15^{\prime \prime}$ (the JCMT beam at $800 \mu \mathrm{m})$, comparable to those of the clumps sampled by this study. Such studies often infer hour-glass shaped fields centered on the condensation, consistent with theoretical treatments and the idea of an originally large scale ordered field that is tied to and that is being concentrated along with the collapsing cloud/subclump. Non-zero polarization values toward the centralmost or "pinch" areas imply coherence even in the smallest observable regions. Recent polarization studies suggest a few cases of sharp field reversals, speculated to occur at the boundary of the dusty stellar disk. We are, however, insensitive to any changes that may occur at or within the dusty stellar disk since the gas densities there are far in excess of that being sampled here.

We concede that the Sgr B, the active starforming region in the Galactic center, is perhaps the one case where we may be failing to detect the field due to sign reversals in the beam. In a Hi Zeeman line study performed with the VLA (effective resolution of $10^{\prime \prime}-20^{\prime \prime}$ ), Crutcher et al. (1996a) detected a line-of-sight field of $B_{\|}=-0.5 \mathrm{mG}$ that varies by about a factor of $\sim 50 \%$ across the $1^{\prime}$-diamter complex. Our failure to detect a field toward the Sgr B2 region $\left(B_{\|}<1.8 \mathrm{mG}\right)$ is not particularly surprising given that this is the one instance where the emission from the dense gas almost certainly fills our entire $63^{\prime \prime}$ beam. The extremely large linewidths observed from some molecules toward the Sgr B region $\left(\sim 60 \mathrm{~km} \mathrm{~s}^{-1}\right)$ reflect tremendous inputs of mechanical energy into the gas by means of multiple outflows, expanding shells, etc, and thus great potential for modification of the field.

\subsection{Summary}

Much effort has been expended over the last decade to measure the magnetic flux to mass (or column density) ratio in dark clouds. The relatively diffuse clouds $\left(<10^{3} \mathrm{~cm}^{-3}\right)$, or cloud envelopes traced by $\mathrm{OH}$ (1665 and $1667 \mathrm{MHz}$ ) and $\lambda 21 \mathrm{~cm}$ Hi line emission, appear to be critical or at least approximately so in terms of support by their $B$ fields against collapse (Roberts et al. 1995; 
Troland et al. 1995; Crutcher et al. 1993; Goodman et al. 1989). The matter is potentially different toward the moderate density clumps $\left(n_{\mathrm{H}_{2}}<\sim 10^{4} \mathrm{~cm}^{-3}\right)$, where in the handful of sources sampled thus far, the majority of them (Ori-KL and S106, Crutcher et al. 1996b; B1, Crutcher et al. 1993; $\rho$ Oph, Troland et al. 1996; SgrB2(N), Crutcher et al. 1996a) have line of sight fields, $B_{\|}$, that on the average fall a factor of $\sim 3$ or so below the critical limit. With the stated uncertainties in mind, we maintain that the observed $B_{\|}$field limits determined in this study add weight to that implied by the earlier handful of data points, that the magnetic fields in dense clouds are supercritical or not capable of supporting those clouds against collapse.

There is a great need for more Zeeman observations, coupling to high gas densities, to assess the role the fields play in the late stages of the stellar formation process. Few radicals are known to be suitable and while Crutcher et al. (1996b, 1999) explored the use of CN, we explored the potential of SO and CCS. Despite using the most sensitive telescope available, a dedicated receiver, and spending many hundreds of hours of observation/integration time, we failed to detect any field via the SO and CCS transitions. It is difficult to forsee any technological advancements in the near future that will allow us to approach the critical sensitivity regieme with SO and CCS Zeeman line splitting observations. The more promising approach appears to be follow-up observations of $\mathrm{CN}$, as is being done by Crutcher et al.

\section{Appendix A: Properties of the CCS radical}

CCS is a linear radical whose electronic ground state is the ${ }^{3} \Sigma$ configuration (Saito et al. 1987), i.e., the projection of the orbital angular momentum onto the molecular axis vanishes, $\Lambda=0$, and spin angular momentum $S=$ 1. Since the electronic orbital angular momentum $\boldsymbol{L}$ is far more strongly coupled to the molecular axis than the spin (at least for the slow molecular rotations considered here), the intrinsic magnetic field due to the molecular rotation is expected to couple the spin magnetic moment to the molecular axis. The limiting case is usually described in terms of Hund's case $b$ (e.g., Townes \& Schawlow 1975). In the case of CCS, however, the dipole-dipole interaction between the two unpaired electrons is unusually large. For small rotational and projected orbital angular momenta, $\boldsymbol{R}$ and $\boldsymbol{\Lambda}$, i.e., small $\boldsymbol{N}(=\boldsymbol{R}+\boldsymbol{\Lambda})$, the intrinsic magnetic field associated with the molecular rotation becomes so small that the spin-spin interaction dominates the coupling of the electronic spin onto the molecular axis. This case is rather described by Hund's case $a$, where the total angular momentum $\boldsymbol{J}$ results from the coupling of $\boldsymbol{\Sigma}$ and $\boldsymbol{N}$; the angular momentum $\boldsymbol{\Sigma}$ is the projection of $\boldsymbol{S}$ onto the molecular axis, and, correspondingly, $\boldsymbol{\Omega}$ is the projection of $\boldsymbol{J}$.
The energy associated with the magnetic dipole interaction of a paramagnetic molecule with an external magnetic field $\boldsymbol{B}$ is given by

$$
\Delta E_{B}=-\boldsymbol{\mu}_{\mathrm{JN}} \boldsymbol{B}
$$

where $\boldsymbol{\mu}_{\mathrm{JN}}$ is the magnetic moment of the CCS radical in state $J_{N}$, associated with the total angular momentum operator $\boldsymbol{J}$

$$
\boldsymbol{\mu}_{\mathrm{JN}}=-g_{\mathrm{JN}} \frac{\mu_{\mathrm{B}}}{\hbar} \boldsymbol{J}
$$

Generally, $\boldsymbol{\mu}_{\mathrm{JN}}$ results from individual contributions (e.g., Judd 1975),

$\boldsymbol{\mu}_{\mathrm{JN}}=-\frac{\mu_{\mathrm{B}}}{\hbar}\left(g_{L} \boldsymbol{L}+g_{S} \boldsymbol{S}\right)+\frac{\mu_{\mathrm{n}}}{\hbar}\left(g_{I} \boldsymbol{I}+g_{R} \boldsymbol{R}\right)$

where $\mu_{\mathrm{B}}$ is the Bohr magneton, $\mu_{\mathrm{n}}$ is the nuclear magneton, $\boldsymbol{I}$ is the nuclear spin, $\boldsymbol{L}$ is the electronic orbital and $\boldsymbol{N}$ the total orbital angular momentum. The $g$-factors are defined as usual. For CCS in its ground state configuration the terms involving $\boldsymbol{I}$ and $\boldsymbol{N}$ can be neglected since $\mu_{\mathrm{n}} \ll \mu_{\mathrm{B}}$. Therefore, the magnetic moment $\boldsymbol{\mu}_{\mathrm{JN}}$ can be expressed by

$\boldsymbol{\mu}_{\mathrm{JN}}=-\frac{\mu_{\mathrm{B}}}{\hbar}\left(g_{L} \boldsymbol{L}+g_{S} \boldsymbol{S}\right)$

to an excellent approximation. The $J_{N}=0_{1}$ level has a vanishing magnetic moment because of $J=0$. The rotational ground state can thus not split into magnetic sublevels due to an external magnetic field. Taking the quantization axis along the direction of $\boldsymbol{B}$ the splitting energy of other rotational levels $J_{N}$ due to the Zeeman effect is given by

$\Delta E_{B}=g_{\mathrm{JN}} \mu_{\mathrm{B}} M_{\mathrm{JN}} B$

where $M_{\mathrm{JN}}$ is the corresponding magnetic quantum number and $B$ is the magnetic field strength.

\section{A.1. The $g_{J N}$-factor}

Since the lower rotational states can be approximated by Hund's case $a$, we designate eigenvectors by $\mid \Sigma \Omega S J>$. Because of $S=1$ and $\Lambda=0$ in the $J=1$ state, $\Sigma=$ $+1,0,-1$, and $\Omega=|\Lambda+\Sigma|=0,1$. Hence, there are three corresponding eigenvectors: $|1111>| 0011>$, and $\mid-1111>$.

For any linear molecule in the ${ }^{3} \Sigma$ configuration, the Hamiltonian is given by (Gordy \& Cook 1984)

$$
\boldsymbol{H}=h\left(B_{0} \boldsymbol{N}^{2}+2 D_{0} \boldsymbol{N}^{4}+\frac{2}{3}\left(\lambda_{0}+\lambda_{1} \boldsymbol{N}^{2}\right)\left(3 S_{Z}^{2}-\boldsymbol{S}^{2}\right)+\gamma \boldsymbol{N} \boldsymbol{S}\right)
$$

where (in the case of CCS) $B_{0}(=6477.74952 \mathrm{MHz})$ is the rotation constant, $D_{0}(=1.72704 \mathrm{kHz})$ is the centrifugal stretching, $\lambda_{0}(=97193.79 \mathrm{MHz})$ is the spin-spin coupling constant, $\lambda_{1}(=27.14 \mathrm{kHz})$ is the centrifugal correction, and $\gamma(=-14.645 \mathrm{MHz})$ is the spin-rotation coupling constant (Saito et al. 1987). 
In order to express the above Hamiltonian in a form that is suitable for case $a$ eigenvectors, we notice that in case $b$

$\boldsymbol{J}=\boldsymbol{N}+\boldsymbol{S}$,

which is equivalent to

$\boldsymbol{N S}=(\boldsymbol{J}-\boldsymbol{S}) \boldsymbol{S}=\boldsymbol{J} \boldsymbol{S}-\boldsymbol{S}^{2}$

so that

$\boldsymbol{H}=h B_{0}\left(\boldsymbol{J}^{2}+\boldsymbol{S}^{2}-2 \boldsymbol{J} \boldsymbol{S}+h D_{0}\left(\boldsymbol{J}^{2}+\boldsymbol{S}^{2}-2 \boldsymbol{J} \boldsymbol{S}\right)^{2}+\right.$

$\frac{2 h}{3}\left(\lambda_{0}+\lambda_{1}\left(\boldsymbol{J}^{2}+\boldsymbol{S}^{2}-2 \boldsymbol{J} \boldsymbol{S}\right)\right)\left(3 S_{Z}^{2}-\boldsymbol{S}^{2}\right)+h \gamma\left(\boldsymbol{J} \boldsymbol{S}-\boldsymbol{S}^{2}\right)$.

The operator product $\boldsymbol{J} \boldsymbol{S}$ will be expressed in terms of the ladder operators in order to apply eigenvalue equations later on,

$\boldsymbol{J} \boldsymbol{S}=\frac{1}{2}\left(\boldsymbol{J}_{+} \boldsymbol{S}_{-}+\boldsymbol{J}_{-} \boldsymbol{S}_{+}\right)+\boldsymbol{J}_{Z} \boldsymbol{S}_{Z}$

using

$\boldsymbol{J}_{ \pm}=\boldsymbol{J}_{X} \pm i \boldsymbol{J}_{Y}$

$\boldsymbol{S}_{ \pm}=\boldsymbol{S}_{X} \pm i \boldsymbol{S}_{Y}$

The state vectors for $\boldsymbol{H}$ can now be formed from linear combinations of the eigenvectors,

$\left|\psi_{n}>=\sum_{i} c_{i}^{n}\right| \Sigma \Omega S J>$.

For the solution of Schrödinger's equation,

$\boldsymbol{H}\left|\psi_{n}>=E_{n}\right| \psi_{n}>$,

the following eigenvalue equations have to be considered (e.g., Hougen 1970),

$$
\begin{aligned}
& \boldsymbol{J}^{2}\left|\Sigma \Omega S J>=\hbar^{2} J(J+1)\right| \Sigma \Omega S J> \\
& \boldsymbol{S}^{2}\left|\Sigma \Omega S J>=\hbar^{2} S(S+1)\right| \Sigma \Omega S J> \\
& \boldsymbol{J}_{Z}|\Sigma \Omega S J>=\hbar \Omega| \Sigma \Omega S J> \\
& \boldsymbol{S}_{Z}|\Sigma \Omega S J>=\hbar \Sigma| \Sigma \Omega S J>
\end{aligned}
$$

and

$$
\begin{aligned}
& \boldsymbol{S}_{+}|\Sigma \Omega S J>=\hbar \sqrt{(S-\Sigma)(S+\Sigma+1)}| \Sigma+1 \Omega S J> \\
& \boldsymbol{J}_{+}|\Sigma \Omega S J>=\hbar \sqrt{(J+\Omega)(J-\Omega+1)}| \Sigma \Omega-1 S J> \\
& \boldsymbol{S}_{-}|\Sigma \Omega S J>=\hbar \sqrt{(S+\Sigma)(S-\Sigma+1)}| \Sigma-1 \Omega S J> \\
& \boldsymbol{J}_{-}|\Sigma \Omega S J>=\hbar \sqrt{(J-\Omega)(J+\Omega+1)}| \Sigma \Omega+1 S J>.
\end{aligned}
$$

With those equations we obtain the matrix elements listed in Table A.1. The solutions to Schrödinger's equation in matrix form

$<\psi_{m}|\boldsymbol{H}| \psi_{n}>-E_{n} \delta_{m n}=0$

can be found from the secular equation

$\operatorname{det}\left(H_{m n}-E_{n} \delta_{m n}\right)=0$.
The solutions are the energy eigenvalues which correspond to three energy levels in the $J=1$ state, $N=0,1,2$, as shown in Fig. 2 of Saito et al. (1987),

$$
\begin{aligned}
& E_{0}=h(-104.964 \mathrm{GHz}) \\
& E_{1}=h(+77.751 \mathrm{GHz}) \\
& E_{2}=h(+79.583 \mathrm{GHz}) .
\end{aligned}
$$

The smallest of those three energy eigenvalues corresponds to the energy level $J_{N}=1_{0}$.

In order to find the wavefunction for this energy eigenstate, the coefficients $c_{\mathrm{i}}^{0}$ from the linear combination (Eq. (A.5)) must be determined. Rewriting Eq. (A.5) as (omitting the superscript 0 in the following)

$\mid \psi_{0}>=\left(\begin{array}{l}c_{-1} \\ c_{0} \\ c_{+1}\end{array}\right)$

Eq. (A.15) yields

$$
\begin{aligned}
182.730054 c_{-1}-12.970087 c_{0}-0.000007 c_{+1} & =0 \\
-12.970160 c_{-1}+1.312410 c_{0}-12.970160 c_{+1} & =0 \\
-0.000007 c_{-1}-12.970087 c_{0}+182.730054 c_{+1} & =0 .
\end{aligned}
$$

where the matrix elements in Table 1 were used. Both the first and third equation give $c_{-1}=c_{+1}$, and consequently

$\frac{c_{+1}}{c_{0}}=0.07098$.

Hence the above system of equations is redundant, and a normalization is introduced,

$c_{-1}^{2}+c_{0}^{2}+c_{+1}^{2}=1$,

which allows to determine the individual coefficient to

$c_{0}=\left(1+2\left(\frac{c_{+1}}{c_{0}}\right)^{2}\right)^{-\frac{1}{2}}=0.99500$

$c_{+1}=c_{-1}=\left(\frac{1-c_{0}^{2}}{2}\right)^{\frac{1}{2}}=0.07062$.

The wavefunction for the $J_{N}=1_{0}$ level can now be given explicitely,

$\mid \psi_{0}>=$

$0.07062|1111>+0.99500| 0011>+0.07062 \mid-1111>$.

In order to find the $g_{\mathrm{JN}}$-factor for the $J_{N}=1_{0}$ level, Eqs. (A.1) and (A.2) were multiplied by $\boldsymbol{J}$ to obtain

$\frac{\mu_{\mathrm{B}}}{\hbar}\left(g_{L} \boldsymbol{J} \boldsymbol{L}+g_{S} \boldsymbol{J} \boldsymbol{S}\right)=g_{\mathrm{JN}} \frac{\mu_{\mathrm{B}}}{\hbar} \boldsymbol{J}^{2}$.

The corresponding expectation value in state $\left|\psi_{0}\right\rangle$ is found by using Eqs. (A.7) and (A.16),

$g_{L}<\psi_{0}|\boldsymbol{J} \boldsymbol{L}| \psi_{0}>+g_{S}<\psi_{0}|\boldsymbol{J} \boldsymbol{S}| \psi_{0}>=$

$g_{\mathrm{JN}}<\psi_{0}\left|\boldsymbol{J}^{2}\right| \psi_{0}>=g_{\mathrm{JN}} \hbar^{2} J(J+1)$. 
Table A.1. Matrix elements

\begin{tabular}{|c|c|c|}
\hline$<1111|\boldsymbol{H}| 1111>$ & $=$ & $h\left(2 B_{0}-8 D_{0}+\frac{2}{3} \lambda_{0}+\frac{4}{3} \lambda_{1}-\gamma\right)=h(77.766026 \mathrm{GHz})$ \\
\hline$<1111|\boldsymbol{H}| 0011>$ & $=$ & $h\left(-2 B_{0}+12 D_{0}+\frac{8}{3} \lambda_{1}+\gamma\right)=h(-12.970087 \mathrm{GHz})$ \\
\hline$<1111|\boldsymbol{H}|-1111>$ & $=$ & $h\left(-4 D_{0}\right)=h(-7 \mathrm{kHz})$ \\
\hline$<0011|\boldsymbol{H}| 1111>$ & $=$ & $h\left(-2 B_{0}+12 D_{0}-\frac{4}{3} \lambda_{1}+\gamma\right)=h(-12.970160 \mathrm{GHz})$ \\
\hline$<0011|\boldsymbol{H}| 0011>$ & $=$ & $h\left(4 B_{0}-24 D_{0}-\frac{4}{3} \lambda_{0}-\frac{16}{3} \lambda_{1}-2 \gamma\right)=h(-103.651618 \mathrm{GHz})$ \\
\hline$<0011|\boldsymbol{H}|-1111>$ & $=$ & $h\left(-2 B_{0}+12 D_{0}-\frac{4}{3} \lambda_{1}+\gamma\right)=h(-12.970160 \mathrm{GHz})$ \\
\hline$<-1111|\boldsymbol{H}| 1111>$ & $=$ & $h\left(-4 D_{0}\right)=h(-7 \mathrm{kHz})$ \\
\hline$<-1111|\boldsymbol{H}| 0011>$ & $=$ & $h\left(-2 B_{0}+12 D_{0}+\frac{8}{3} \lambda_{1}+\gamma\right)=h(-12.970087 \mathrm{GHz})$ \\
\hline$<-1111|\boldsymbol{H}|-1111>$ & $=$ & $h\left(2 B_{0}-8 D_{0}+\frac{2}{3} \lambda_{0}+\frac{4}{3} \lambda_{1}-\gamma\right)=h(77.766026 \mathrm{GHz})$ \\
\hline
\end{tabular}

The evaluation of the expectation value for $\boldsymbol{J} \boldsymbol{L}$ in state $\mid \psi_{0}>$ requires eigenvalue equations for $\boldsymbol{L}_{\boldsymbol{X}}, \boldsymbol{L}_{\boldsymbol{Y}}$ and $\boldsymbol{L}_{\boldsymbol{Z}}$. In analogy to the eigenvalue equations for the spin angular momentum, corresponding equations for the electronic orbital angular momentum can be found substituting $\boldsymbol{S}$ by $\boldsymbol{L}, S$ by $L$ and $\Sigma$ by $\Lambda$ in Eqs. (A.7) through (A.14). (Hougen 1970). Obviously, the operators $\boldsymbol{L}_{ \pm}$and $\boldsymbol{L}_{\boldsymbol{Z}}$ have non-vanishing matrix elements only if the selection rule $\Delta \Lambda= \pm 1$ is satisfied, and $\Lambda \neq 0$, respectively. Neither of these requirements is fulfilled for the expectation value of $\boldsymbol{J} \boldsymbol{L}$ in state $\left|\psi_{0}\right\rangle$, so that $\left\langle\psi_{0}|\boldsymbol{J} \boldsymbol{L}| \psi_{0}\right\rangle=0$, hence

$g_{\mathrm{JN}}=\frac{g_{S}}{J(J+1)} \frac{1}{\hbar^{2}}<\psi_{0}|\boldsymbol{J} \boldsymbol{S}| \psi_{0}>$.

Using Eq. (A.4) and the eigenvalue Eqs. (A.9)-(A.14),

$<\psi_{0}|\boldsymbol{J} \boldsymbol{S}| \psi_{0}>=\hbar^{2}\left(2 c_{+1}^{2}+4 c_{0} c_{+1}\right)=\hbar^{2}$.

From Eq. (A.17) and recalling that $g_{S}=2.0023$, the $g_{\mathrm{JN}^{-}}$ factor for $J_{N}=1_{0}$ level is finally determined to be

$g_{10}=0.29$.

\section{References}

André, Ph., Martin-Pintado, J., Depois, D., \& Montmerle, T. 1990, A\&A, 236, 180

André, Ph., Ward-Thompson, D., \& Barsony, M. 1993, ApJ, 406, 122

Bally, J. 1982, ApJ, 261, 558

Basu, S., \& Mouschovias, T. Ch. 1994, ApJ, 432, 720

Basu, S., \& Mouschovias, T. Ch. 1995, ApJ, 452, 386

Blake, G. A., Sutton, E. C., Masson, C. R., \& Phillips, T. G. 1987, ApJ, 315, 621

Blandford, R. D., \& Payne, D. G. 1982, MNRAS, 199, 883

Carlberg, R. G., \& Pudritz, R. E. 1990, MNRAS, 247, 353

Cesaroni, R., \& Walmsley, C. M. 1991, A\&A, 241, 537

Cesaroni, R., Walmsley, C. M., \& Churchwell, E. 1992, A\&A, 256,618

Cesaroni, R., Walmsley, C. M., Kömpe, C., \& Churchwell, E. 1991, A\&A, 252, 278

Chandler, C. J., Moore, T. J. T., Mountain, C. M., \& Yamashita, T. 1993, MNRAS, 261694

Chernin, L., \& Masson, C. 1992, ApJL, 396, L35

Chernin, L., \& Masson, C. 1993, ApJL, 403, L21

Cox, P., Walmsley, C. M., \& Güsten, R. 1989, A\&A, 209, 382 Crutcher, R. M. 1999, ApJ, 520, 706

Crutcher, R. M., Roberts, D. A., Mehringer, D., \& Troland, T. H. 1996a, ApJ, 462, 79
Crutcher, R. M., Troland, T. H., Goodman, A. A., et al. 1993, ApJ, 407, 175

Crutcher, R. M., Troland, T. H., Lazareff, B., \& Kaz̀es, I. 1996b, ApJ, 456, 217

Crutcher, R. M., Troland, T. H., Lazareff, B., Paubert, G. \& Kaz̀es, I. 1999, ApJ, 514, L121

Dickel, H. R., Dickel, J. R., Wilson, W. J., \& Werner, M. W. 1980, ApJ, 237, 711

Dickel, H. R., \& Goss, W. M. 1987, A\&A, 185, 271

Fiebig, D. 1990, Ph.D. Thesis, University of Bonn

Guilloteau, S., Stier, M., \& Downes, D. 1984, A\&A, 126, 10

Gómez, Y., Rodriguez, L. F., Garay, G., \& Moran, J. M. 1991, ApJ, 377, 519

Goodman, A. A., Crutcher, R. M., Heiles, C., Myers, P. C., \& Troland, T. H. 1989, ApJ, 338, L61

Gordy, W., \& Cook, R. L. 1984, Microwave Molecular Spectra, 3 ed. (Interscience Publ., New York)

Gottlieb, C. A., Gottlieb, E. W., Litvak, M. M., Ball, J. A., \& Penfield, H. 1978, ApJ, 219, 77

Graasdalen, G. L., Strom, K. M., \& Strom, S. E. 1973, ApJL, $184, \mathrm{~L} 53$

Green, S. 1994, ApJ, 434, 188

Güsten, R., Fiebig, D., \& Uchida, K. I. 1994, A\&A, 286, L51

Hasegawa, T. I., Mitchell, G. F., Matthews, H. E., \& Tacconi, L. 1994, ApJ, 426, 215

Hartmann, L., \& McGregor, K. B. 1982, ApJ, 259, 180

Harquist, T. W., Dalgarno, A., \& Oppenheimer, M. 1980, ApJ, 236,182

Hauschildt, H., Güsten, R., Phillips, T. G., et al. 1993, A\&A, 273, L73

Helmich, F. P., Jansen, D. J., de Grauuw, T. H., Groesbeck, T. D., \& van Dishoek, E. F. 1994, A\&A, 283, 626

Ho, P. T. P., Terebey, S., \& Turner, J. L. 1994, ApJ, 423, 320

Ho, P. T. P., \& Haschick, A. D. 1986, ApJ, 304, 501

Holland, W. S., Greaves, J. S., Ward-Thompson, D., \& Andre, Ph. 1995, Ap\&SS, 224, 487

Hougen, J. T. 1970, Monograph \#115, National Bureau of Standards, Washington, USA

Hüttemeister, S., Wilson, T. L., Henkel, C., \& Mauersberger, R. 1993, A\&A, 276, 445

Hüttemeister, S., Wilson, T. L., Mauersberger, R., et al. 1995, A\&A, 294, 667

Jones, K. N., Field, D., Gray, M. D., \& Walker, R. N. F. 1994, A\&A, 288, 581

Judd, B. R. 1975, Angular Momemtum Theory for Diatomic Molecules (Academic Press, New York)

Keto, E. R., Ho, P. T. P., \& Haschick, A. D. 1987, ApJ, 318, 712

Königl, A., \& Ruden, S. 1993, in Protostars and Planets III, ed. M. Matthews, \& E. Levy (Univ. Arizona Press, Tucson), 641 
Larson, R. B. 1981, MNRAS, 194, 809

Lightfoot, J. F., Deighton, D. W., Furniss, I., et al. 1984, MNRAS, 208, 197

Mangum, J. G., Wootten, A., \& Mundy, L. G. 1991, ApJ, 378, 576

Mangum, J. G., Wootten, A., \& Mundy, L. G. 1992, ApJ, 388, 467

Martin-Pintado, J., Bachiller, R., \& Fuente, A. 1992, A\&A, 254,315

Martin-Pintado, J., Wilson, T. L., Gardner, F. F., \& Henkel, C. 1983, A\&A, 117, 145

Mckee, C. F., Zweibel, E. G., Goodman, A. A., \& Heiles, C. 1993, in Protostars and Planets III, ed. M. Matthews, \& E. Levy (Univ. Arizona Press, Tucson)

Myers, P. C., \& Goodman, A. A. 1988, ApJ, 329, 392

Minchin, N. R., \& Murray, A. G. 1994, A\&A, 286, 579

Minchin, N. R., Ward-Thompson, D., \& White, G. J. 1995, A\&A, 298, 894

Mitchell, G. F. 1984, ApJS, 54, 81

Mouschovias, T. Ch., Paleologou, E. V., \& Fiedler, R. A. 1985, ApJ, 291, 772

Mouschovias, T. Ch., \& Spitzer, L. Jr. 1976, ApJ, 210, 326

Moriarty-Schieven, G. H., Snell, R. L., \& Hughes, V. A. 1989, ApJ, 347, 358

Nakano, T. 1990, MNRAS, 242, 535

Neufeld, D. A., \& Dalgarno A. 1989, ApJ, 340, 869

Norman, C., \& Silk, J. 1980, ApJ, 238, 158

Oldham, P. G., Griffin, M. J., Richardson, K. J., \& Sandell, G. 1994, A\&A, 284, 559

Padin, S., et al. 1989, ApJ, 337, L45

Persson, S. E., Geballe, T. R., Simon, T., Lonsdale, C. J., \& Baas, F. 1981 ApJL, 251, L85

Plambeck, R. L., Wright, M. C. H., Welch, W. J., et al. 1982, ApJ, 259, 617

Pudritz, R. E., \& Norman, C. A. 1983, ApJ, 274, 677

Pudritz, R. E., \& Norman, C. A. 1986, ApJ, 301, 571

Reid, M. J., Myers, P. C., \& Bieging, J. H. 1987, ApJ, 312, 830

Roberts, D. A., Crutcher, R. M., \& Troland, T. H. 1995, ApJ, 442,208

Richardson, K. J., Sandell, G., White, G. J., Duncan, W. D., \& Krisciunas, K. 1989, A\&A, 221, 95
Saito, S., Kawaguchi, K., Yamamoto, S., et al. 1987, ApJL 317, L 115

Schmid-Burgk, J., \& Muders, D. 1994, in Stellar and Circumstellar Astrophysics, ed. G. Wallerstein, \& A. Noriega-Crespo, ASP Conf. Ser., 57, 74

Scoville, N. Z., Sargent, A. I., Sanders, D. B., et al. 1986, ApJ, 303, 416

Snell, R. L., Scoville, N. Z., Snaders, D. B., \& Erickson, N. R. 1984, ApJ, 284, 176

Stutzki, J., Genzel, R., Harris, A. I., Herman, J., \& Jaffe, D. T. 1988, ApJ, 330, L125

Tieftrunk, A. R., Wilson, T. L., Steppe, H., et al. 1995, A\&A, 303, 901

Tomisaka, K., Ikeuchi, S., \& Nakamura, T. 1990, ApJ, 362, 202

Townes, C. H., \& Schawlow, A. L. 1975, Microwave Spectroscopy (Dover Publ. Inc., New York)

Troland, T. H., Crutcher, R. M., Goodman, A. A., et al. 1996, ApJ, 471, 302

Ungerechts, H., Winnewisser, G., \& Walmsley, C. M. 1986, A\&A, 157, 207

Vogel, S. N., Genzel, R., \& Palmer, P. 1987, ApJ, 316, 243

Wadiak, E. J., Rood, R. T., Wilson, T. L., \& Johnston, K. J. 1985, ApJ, 295, 43

Wilson, T. L., Johnston, K. J., \& Mauersberger, R. 1991, A\&A, 251, 220

Wilson, T. L., \& Mauersberger, R. 1990, A\&A, 239, 305

Wink, J. E., Duvert, G., Guilloteau, S., et al. 1994, A\&A, 281, 505

Wright, M. C. H., Dickel, H. R., \& Ho, P. T. P. 1984, ApJ, 281, L71

Wright, M. C. H., Plambeck, R. L., \& Wilner, D. J. 1996, ApJ, 469,216

Wynn-Williams, C. G., Becklin, E. E., \& Beugebauer, G. 1972, MNRAS, 160, 1

Zhou, S., Butner, H. M., Evans, N. J. II, et al. 1994, ApJ, 428, 219

Zhou, S., Evans, N. J. II, Güsten, R., Mundy, L. G., \& Kutner, M. L. 1991, ApJ, 372, 518

Zuckerman, B., \& Evans, N. J. II 1974, ApJL, 192, L149 